\title{
Paradigms and paradoxes: fractional electron charge
}

\author{
Istvan Hargittai ${ }^{1}$
}

Accepted: 11 December 2021 / Published online: 20 December 2021

(c) The Author(s) 2021

\begin{abstract}
There is hardly a generic connection between the partial atomic charges, a useful concept in chemistry, and the "fractionalization" of the electron accomplished under extreme experimental conditions in solid samples. Nonetheless, there is a relationship on a philosophical level. There is no information of who first introduced the concept of partial atomic charges in chemistry. In contrast, the physicists whose experiment turned the electron into excitations carrying a partial charge and whose theory provided the interpretation received the Nobel Prize for their discoveries.
\end{abstract}

Keywords Partial atomic charges · Fractionalization of electrons · Quantum theory of solids · Quantum fluids · Horst L. Störmer · Daniel C. Tsui · Robert B. Laughlin · Bell Labs

Chemical intuition has played a most fruitful role in the development of chemistry. For example, it was a revolutionary thought to interpret chemical happening with the motion of electron at the time when there was hardly any experimental evidence of such motion during a chemical reaction. When those short, bent arrows in chemical equations first appeared in publications, it was more fantasy than fact. According to some anecdotes, the typesetters in the press arranged those arrows to fit the available space rather than ascribing true meaning to them-and, apparently, the authors did not protest. When the lower case Greek deltas appeared attached to positive or negative charges, $\delta+$ and $\delta-$, they meant to indicate partial rather than full charges on specific atoms. Nobody seems to have bothered to contemplate whether it would be possible to have in reality a fraction of the electron's negative charge, that is, a fraction of an electron. Eventually, with probability density distributions, partial electrons gained meaning-in the sense of probabilities-and they could also be visualized: they could be constructed from experimental, X-ray diffraction data, and computed and projected as maps of charge density distribution.

This had already been achieved in chemistry when the news about the 1998 physics Nobel Prize was announced,

Istvan Hargittai

stuceditor@gmail.com

1 Budapest University of Technology and Economics, Budapest 1521, Hungary awarded to three physicists (Fig. 1): Robert B. Laughlin (b. 1950 in Visalia, CA) of Stanford University, earlier at Livermore National Laboratory and at MIT; Horst L. Störmer (b. 1949 in Frankfurt, Germany) of Columbia University, earlier at Bell Labs; and Daniel C. Tsui (b. 1939 in Henan, China) of Princeton University, earlier at Bell Labs; received it jointly. The citation read as follows: "for their discovery of a new form of quantum fluid with fractionally charged excitations." The two experimental physicists, Störmer and Tsui, conducted their experiments at Bell Labs. They used powerful magnetic fields and extremely low temperatures. The theoretical physicist Laughlin did his relevant work at Livermore, and interpreted the puzzling experimental observations. He concluded that under the extreme conditions, the electrons condensed into a quantum fluid, which could be related to the quantum fluid characteristic of superfluidity in liquid helium. A remarkable property of this electron quantum fluid is that if one electron is added to it, the fluid will be "excited," yielding fractionally charged "quasiparticles." Demonstrating the presence of fractional charges of these quasiparticles explained Störmer and Tsui's puzzling observations. The preceding description calls for further reading of the available literature, and, for a good starting point, their respective Nobel lectures are recommended (here are the print sources [1-3] and the lectures are also available on the site of the Nobel Foundation [4]). Here, it suffices to convey the notion of the existence of fractional charges under some extreme experimental conditions. What the chemists have intuited appeared to be a useful possibility 

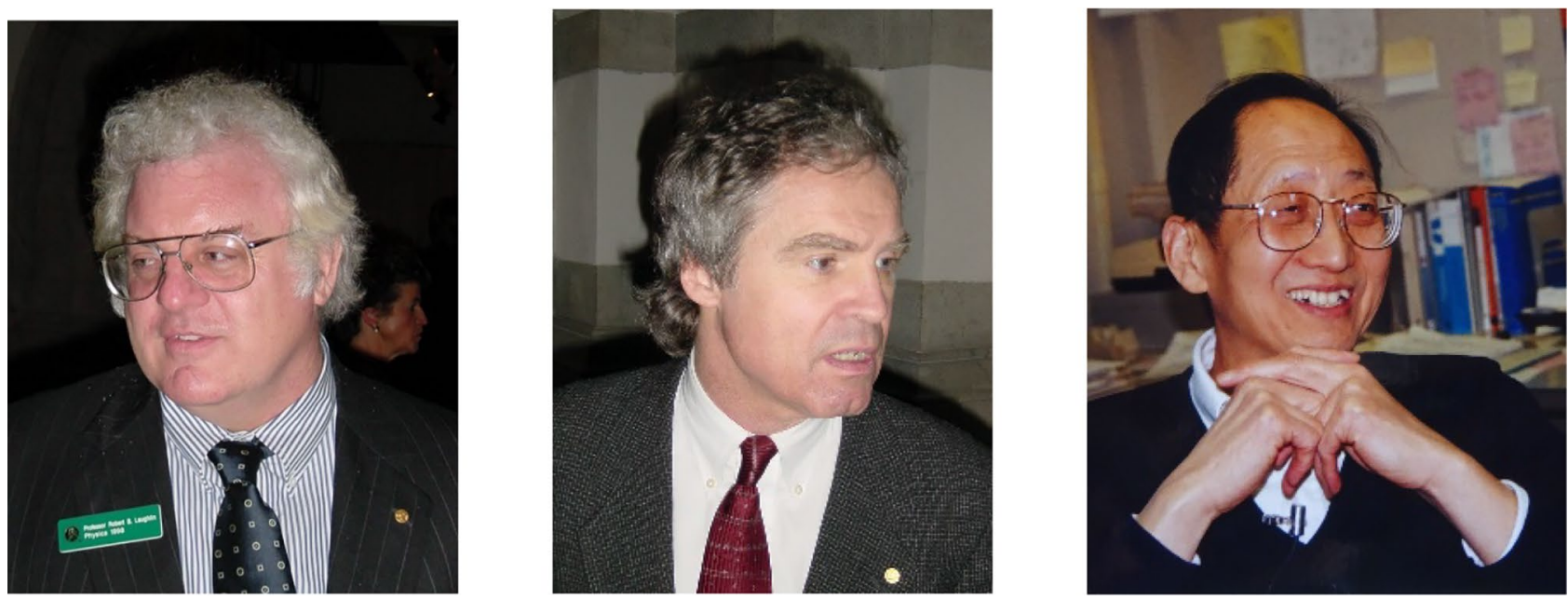

Fig. 1 from left to right, Robert B. Laughlin, 2001, Stockholm; Horst L. Störmer, 2001, Stockholm; and Daniel C. Tsui, 1999, Princeton (all three images by I. Hargittai)

in the chemical equations even though hardly related to the fractional charges of the quasiparticles mentioned above.

It appears farfetched to discuss the partial atomic charges intuited in chemistry and the composite particles originating from electrons and carrying partial charges. Yet, there is a sensation of their connection at a philosophical level. This was also what Daniel C. Tsui implied when I talked with him many years ago about his and Störmer's experiment at Bell Labs. He first commented about "the artificiality of science being divided into different fields such as physics, chemistry, biology. Phil Anderson used the word of cross-reference and the analogy of how many different crafts go into building a cathedral. You are asking me about our experiment. At that time, it was just a singular experiment. We knew that it was something new and something interesting but for its significance, it takes time to gradually appreciate it. It is related to a lot of beautiful ideas and concepts and you see in it also the clear emergence of physical reality. The most recent example has been the better understanding of composite particles. It has also contributed to various field theories. One can literally imagine now how the electron binds to magnetic fluxes, forming a new particle. By forming a new composite particle, the statistics also changes, and instead of a fermion it becomes a boson. It also has relevance to the interaction of electrons, which is so important in chemistry." [5].

Tsui made it also clear that it was not really the electron charge that changed; rather, the charge of the excitation emerging from the interactions among the electrons was a fraction of the electron charge: "The electron entity has still the same charge. All the electrons move together in this quantum fluid in a correlated manner and the smallest excitation carries a charge that is a third of the electron charge. It does not mean that the individual electrons carry a third of the charge. The excitation creates a new particle in a sense whose charge is a fraction of the electron charge. You have to consider the whole system. You do something to the system and the result of the excitation is the new particles whose movement you characterize as the particle carrying a third of the electron charge. You make this conclusion from the behavior of the new particle." [5].

Störmer and Tsui's experiments happened in 1982 at Bell Labs. It was a great research venue where its associates were encouraged to carry out curiosity-driven experiments, and as their results, spectacular discoveries emerged. Suffice it to mention Clinton J. Davisson's experiment together with his associate, Lester H. Germer, in 1927, when they discovered electron diffraction by crystals. It was the cradle of brand new techniques in physics, chemistry, and materials science. Perhaps, its significance was even higher as it provided experimental evidence for the wave-particle, dual, nature of matter demonstrated theoretically only a few years before by Louis de Broglie. In that case, theory was followed by experiment. In case of the partial charges by excitations in the electron fluid, theory followed the experiment. In both cases, Nobel Prizes followed the discoveries. Davisson and Germer worked for Bell labs at what used to be their Manhattan campus, which no longer exists except for a hardly visible memorial tablet commemorating the Davisson-Germer experiment [6]. Störmer and Tsui worked at Bell Labs in New Jersey. Soon after their milestone experiment, they left for academia; Störmer became a professor at the physics department of Columbia University and Tsui a professor at the department of electrical engineering of Princeton University. The story illustrates the wonderful synergy of industry and academia; theory and experiment; and even the internationality of science.

As for the significance of the Störmer-Tsui-Laughlin discovery, Philip W. Anderson put it beautifully, a mere one year before the announcement of the 1998 Nobel Prizes. He 
wrote about the new development in the theory of electrons in solids and used the expression of the "fractionalization" of the electron. He emphasized the importance of "the discovery of particles that behave as though the electron had broken apart into three or five or more pieces each containing one-third or one-fifth of its charge ..." [7].

Returning to the chemical intuition concerning partial atomic charges, it has been fully vindicated. In the covalent bond of the hydrogen molecule-ion, $\mathrm{H}_{2}{ }^{+}$, one electron is assigned to the two hydrogen atoms, belonging to both, thus, providing stability to their bond. This one-electron bond could be represented by a single dot between the two hydrogen atoms, but it is more realistic physically to indicate the electron density distribution between and about the two nuclei [8]. Hydrogen positions are not very amenable to extract from the electron density distributions determined experimentally by X-ray diffraction. However, the electron density distributions from X-ray diffraction and determined by computation ab initio are consistent with each other. The physics discovery described above need not be considered as evidence for the description developed in chemistry, but it provides a feeling of comfort that the electron need not be considered so indivisible as it used to be. Mere intuition, it no longer is. Even though we do not think of fractionalized electrons and fraction charges, the probability density distributions reflect physical reality, and they are consistent with what used to be an intuited concept.

Acknowledgements I thank Magdolna Hargittai and Joel F. Liebman for valuable comments and suggestions.

Funding Open access funding provided by Budapest University of Technology and Economics.

Open Access This article is licensed under a Creative Commons Attribution 4.0 International License, which permits use, sharing, adaptation, distribution and reproduction in any medium or format, as long as you give appropriate credit to the original author(s) and the source, provide a link to the Creative Commons licence, and indicate if changes were made. The images or other third party material in this article are included in the article's Creative Commons licence, unless indicated otherwise in a credit line to the material. If material is not included in the article's Creative Commons licence and your intended use is not permitted by statutory regulation or exceeds the permitted use, you will need to obtain permission directly from the copyright holder. To view a copy of this licence, visit http://creativecommons. org/licenses/by/4.0/.

\section{References}

1. Störmer HL (1999) The fractional quantum Hall effect. In Frängsmyr T, ed, Les Prix Nobel-The Nobel Prizes 1998. Stockholm: The Nobel Foundation 103-133

2. Tsui DC (1999) Interplay of disorder and interaction in twodimensional electron gas in intense magnetic fields. In Frängsmyr T, ed, Les Prix Nobel-The Nobel Prizes 1998. Stockholm: The Nobel Foundation 138-147

3. Laughlin RB (1999) Fractional quantization. In Frängsmyr T, ed, Les Prix Nobel-The Nobel Prizes 1998. Stockholm: The Nobel Foundation 72-94

4. www.nobelprize.org

5. Hargittai M, Hargittai I (2004) Candid Science IV: conversations with famous physicists. London: Imperial College Press, Chapter 31, Daniel C. Tsui 620-625; actual quotes 622

6. Hargittai I, Hargittai M (2017) New York scientific: a culture of inquiry, knowledge, and learning. New York: Oxford University Press 57-58

7. Anderson PW (1997) When the electron falls apart. Phys Today 50(10):42

8. Pauling L (1960) The nature of the chemical bond and the structure of molecules and crystals: an introduction to modern structural chemistry, 3rd edn. Cornell University Press, Ithaca, NY 19

Publisher's Note Springer Nature remains neutral with regard to jurisdictional claims in published maps and institutional affiliations. 